\title{
Dynamics of water injection in an oil-wet reservoir rock at subsurface conditions: Invasion patterns and pore-filling events
}

\author{
Abdulla Alhosani $\odot,{ }^{*}$ Alessio Scanziani, Qingyang Lin $\odot$, Sajjad Foroughi, \\ Amer M. Alhammadi, Martin J. Blunt, and Branko Bijeljic \\ Department of Earth Science and Engineering, Imperial College London, London SW7 2BP, United Kingdom
}

(Received 10 June 2020; accepted 21 July 2020; published 14 August 2020)

\begin{abstract}
We use fast synchrotron $\mathrm{x}$-ray microtomography to investigate the pore-scale dynamics of water injection in an oil-wet carbonate reservoir rock at subsurface conditions. We measure, in situ, the geometric contact angles to confirm the oil-wet nature of the rock and define the displacement contact angles using an energy-balance-based approach. We observe that the displacement of oil by water is a drainagelike process, where water advances as a connected front displacing oil in the center of the pores, confining the oil to wetting layers. The displacement is an invasion percolation process, where throats, the restrictions between pores, fill in order of size, with the largest available throats filled first. In our heterogeneous carbonate rock, the displacement is predominantly size controlled; wettability has a smaller effect, due to the wide range of pore and throat sizes, as well as largely oil-wet surfaces. Wettability only has an impact early in the displacement, where the less oil-wet pores fill by water first. We observe drainage associated pore-filling dynamics including Haines jumps and snap-off events. Haines jumps occur on single- and/or multiple-pore levels accompanied by the rearrangement of water in the pore space to allow the rapid filling. Snap-off events are observed both locally and distally and the capillary pressure of the trapped water ganglia is shown to reach a new capillary equilibrium state. We measure the curvature of the oil-water interface. We find that the total curvature, the sum of the curvatures in orthogonal directions, is negative, giving a negative capillary pressure, consistent with oil-wet conditions, where displacement occurs as the water pressure exceeds that of the oil. However, the product of the principal curvatures, the Gaussian curvature, is generally negative, meaning that water bulges into oil in one direction, while oil bulges into water in the other. A negative Gaussian curvature provides a topological quantification of the good connectivity of the phases throughout the displacement.
\end{abstract}

DOI: 10.1103/PhysRevE.102.023110

\section{INTRODUCTION}

Multiphase flow in porous structures occurs in many natural and industrial systems such as blood flow [1], the movement of food and water within the intestinal tract of the human body [2], transport in porous membranes [3], carbon dioxide storage in geological aquifers [4,5], and oil recovery from reservoir rocks [6,7]. To understand the dynamics of fluid flow in porous materials, we need to study the processes that control its movement, which occur at the pore scale [8]. At the pore scale, the physics underlying the flow is mainly governed by capillary forces that control the fluid-fluid displacement, which depends on the porous medium geometry and wettability.

According to the wettability of the invading and displaced fluids, the displacement processes are termed drainage or imbibition. This definition applies for strictly hydrophilic or hydrophobic systems. However, many natural and manufactured porous media have a wettability that can be altered through contact with surface-active components of the fluids [9]. The resultant wettability controls a variety of processes from oil

\footnotetext{
*Author to whom correspondence should be addressed: Abdulla.alhosani17@imperial.ac.uk
}

recovery to gas exchange in leaves and the performance of fuel cells and batteries [8-13]. Hence, it is of great importance to study and quantify the dynamic nature of invasion patterns and the associated pore-scale events in porous media with an altered wettability. This is the main objective of our study; we now proceed with a discussion of the dynamics of multiphase flow in porous media.

When a porous medium is conceptualized, its void space is typically represented as a network composed of wide regions, the pores, that are connected together by narrower regions, the throats [8]. This network representation is sufficient to accurately characterize and track the filling sequence during displacement. When a nonwetting phase displaces the wetting phase, the process is called drainage: Here the nonwetting phase advances as a connected front through the pore space, displacing the wetting phase in the pore centers and confining it to wetting layers in the corners of the pore space.

Drainage can be described as an invasion percolation process [14], where the nonwetting phase progresses from pore to pore through the widest available throats. Filling is only possible if the nearest-neighbor pore or throat is already filled. An available throat is a throat adjacent to a pore already filled with the invading phase $[14,15]$. The capillary pressure for displacement $P_{c}$ is defined through the Young-Laplace equation, for throats with a cylindrical cross section of radius 
$r$, by

$$
P_{c}=\frac{2 \sigma \cos \theta}{r},
$$

where $\sigma$ is the interfacial tension between the fluids and $\theta$ is their contact angle.

In this work, the displacement process we will investigate is water injection in an oil-filled system with altered wettability. In such systems, the contact angle is conventionally measured through the denser phase, water, while the capillary pressure is the pressure difference between oil and water $\left(P_{c}=P_{o}-P_{w}\right)$. During water injection, the water pressure increases with time and invasion proceeds in order of decreasing capillary pressure; the events with the largest capillary pressure occur first. We will define a drainagelike process for water injection as one represented by a contact angle greater than $90^{\circ}$, where $\cos \theta$ is negative, and hence the capillary pressure is also negative; the medium is water repellent and water has to have a higher pressure than oil to advance through the pore space. For a medium of constant wettability (contact angle), filling the largest available throats corresponds to a capillary-controlled displacement where the invading fluid progresses through the porous medium at the highest allowable capillary pressure (or lowest absolute value, since the capillary pressure is negative).

As the nonwetting phase (water) passes from a narrow throat into a wider pore, there is a sudden change in the local capillary pressure which results in rapid filling of the invaded pore and possibly further filling of multiple pores and throats downstream of the invaded pore if they can also be invaded at the prevailing water pressure. This fast filling is known as a Haines jump [16]. To enable the displacement in multiple pores during a Haines jump, the nonwetting phase retracts from some throats, which we term Roof snap-off $[8,17,18]$. At the pore scale, the retraction of the nonwetting phase is not a drainage process, but instead is an imbibition event, where now the wetting phase displaces the nonwetting phase. Snap-off can occur either in a pore that has just been invaded by the nonwetting phase, local snap-off, or in another region some distance way, distal snap-off [19].

Imbibition, where the contact angles are less than $90^{\circ}$ and the capillary pressure is positive, is considered a more complex pore-scale process and its dynamics is often dominated by snap-off [20]. Snap-off occurs when the layers of the wetting phase start to swell in a throat, and if the wetting layers touch and coalesce, the throat spontaneously fills with the wetting phase, leading to a disconnection and trapping of the nonwetting phase in the centers of the adjacent pore [20-23]. The trapping of the nonwetting phase by snap-off is favorable for $\mathrm{CO}_{2}$ storage applications, where maximum trapping of $\mathrm{CO}_{2}$ (nonwetting phase) is desired, whereas it is detrimental for oil recovery applications.

The early work of Lenormand et al. [21] provided information on the dynamics of pore filling during drainage in two-dimensional micromodels. The same behavior was also observed by Datta et al. [24] in three dimensions, where drainage was investigated in a pack of sintered glass beads using confocal microscopy. However, it was not until recently that advances in $\mathrm{x}$-ray microtomography have allowed for direct imaging of the rock pore space and the fluids within it [25-28]. Many laboratory-based x-ray microtomography studies have provided detailed descriptions of ganglia or disconnected nonwetting phase clusters at the end of imbibition and drainage processes [29-32]. However, these studies only report end point results at static conditions and hence do not capture the displacement or pore-filling sequence which occur on a much shorter timescale than that required for a single scan (which can take several minutes or hours).

To increase the temporal resolution of imaging, fast synchrotron x-ray microtomography can be used, which captures the pore-scale displacement dynamics on a timescale of seconds to around 1 min [18,19,22,23,33-35]. Berg et al. [18] quantified the number of pores invaded by the nonwetting phase ( $n$-decane) during a Haines jump in a water-wet Berea sandstone. Andrew et al. [19] further investigated interface retraction and snap-off during $\mathrm{CO}_{2}$ injection, drainage, in a water-wet Ketton limestone. Moreover, Rücker et al. [23] characterized the impact of the pore-scale viscous effects on snap-off and coalescence events during imbibition in a sandstone rock. However, to date, all the reported dynamic studies were conducted in water-wet media. The displacement dynamics have not been investigated for water invasion in altered-wettability porous media.

In this study, we use synchrotron x-ray microtomography to visualize the pore-scale dynamics during water injection in a reservoir rock with altered wettability. A previous study investigated the dynamics of water injection into a quarry limestone (Ketton) which had been in contact with crude oil [36]. The experimental conditions, mineralogy (mainly calcite), fluids, and wettability alteration protocol of the study conducted in Ketton are almost identical to our study in a reservoir rock. As we show later, although the geometric contact angles measured in situ are similar in the two cases, the macroscopic manifestation in terms of displacement sequence, energy balance, and capillary pressure are different, emphasizing the importance of the interaction of pore geometry and wettability on displacement. In the Ketton rock, the behavior revealed mixed-wet conditions, defined by the simultaneous filling of both small and large pores during water injection, indicating that the displacement was controlled by both pore geometry and wettability (local contact angles); water invasion was not a drainagelike process [36]. However, reservoir rocks are likely to undergo a more significant wettability alteration when in contact with crude oil [37,38], rendering the surfaces largely oil wet (water repellent or hydrophobic). Moreover, the reservoir sample has a wider distribution of pore sizes than in a quarry Ketton sample.

In this paper, we examine, in situ, (i) wettability and displacement contact angles, (ii) invasion patterns (pore-filling order), (iii) Haines jumps, (iv) snap-off events, (v) fluid saturations, (vi) specific interfacial areas, and (vii) oil-water capillary pressure. We validate the hypothesis that in a highly heterogeneous porous medium with a significant wettability alteration from an original water-wet state, the pore space becomes largely water repellent and that water invasion is a drainage-type invasion percolation process. This work is important since non-water-wet surfaces are ubiquitous in nature, e.g., in deep oil reservoirs, rice leaves, butterfly wings, and human skin, as well as the fact that many surfaces are designed to be fully or partially water repellent to improve 
TABLE I. Thermophysical properties of the oil and water phases at the experimental conditions $\left(8 \mathrm{MPa}\right.$ and $\left.60^{\circ} \mathrm{C}\right)$. The interfacial tension between oil and water was measured at $8 \mathrm{MPa}$ and $60^{\circ} \mathrm{C}$ using the pendant drop method. Data are from NIST [45], engineering toolbox [46], and Jianhua et al. [47].

\begin{tabular}{lcccc}
\hline \hline Fluid & Composition (wt. \%) & $\rho\left(\mathrm{kg} \mathrm{m}^{-3}\right)$ & $\mu\left(\mathrm{mPa} \mathrm{s}^{2}\right.$ & $\sigma\left(\mathrm{mN} \mathrm{m}^{-1}\right)$ \\
water & $80 \%$ deionized $+20 \%$ potassium iodide & 1145.0 & 0.547 & $\sigma_{\text {ow }}=52.1$ \\
oil & $85 \%$ n-decane $+15 \%$ 1-iododecane & 715.2 & 1.088 & \\
\hline \hline
\end{tabular}

their performance, e.g., textiles, medical and cosmetic devices including surgical masks, fuel cells, and catalysts [8-13,3942]. Moreover, these time-dependent data are vital for the validation of pore-scale models of multiphase flow.

\section{MATERIALS AND METHODS}

In this section, we provide details regarding the rock and fluid properties, the methodology followed to establish the reservoir wettability, flow apparatus, image acquisition, and processing. The sample was initially prepared in-house before transporting it to the synchrotron facility for imaging the flooding experiment. All imaging was conducted at the Diamond imaging beamline I13-2 (Diamond Light Source, Harwell campus, Didcot, UK).

\section{A. Rock and fluid properties}

The experiment was conducted on a 3.85-mm-diam, 13.8mm-long cylindrical sample of a carbonate rock, extracted from a large producing hydrocarbon reservoir in the Middle East. The mineralogical composition of the rock consisted mainly of calcite $(96.5 \% \pm 1.9 \%)$, with small amounts of dolomite $(1.5 \% \pm 0.3 \%)$, kaolinite $(1.1 \% \pm 0.2 \%)$, and quartz $(0.8 \% \pm 0.4 \%)$ [43]. The reservoir rock is very heterogeneous with a wide distribution of pore sizes varying from 3.5 to $120 \mu \mathrm{m}$; see Fig. S1 for the pore-size distribution [44]. The measured helium porosity of the sample was $26 \%$, with the macro- and microporosities accounting for $16 \%$ and $10 \%$, respectively. In the context of our work, the macroporosity is defined as that which can be directly resolved in the image at the given resolution; the microporosity is the unresolvable pore space in the image. The pore volume (PV) of the sample corresponding to the total helium porosity was $0.0416 \mathrm{ml}$.

The experimental fluids used to represent the water and oil phases were doped deionized water (20\% wt. potassium iodide) and doped $n$-decane ( $15 \%$ wt. 1 -iododecane), respectively. Doping provided a distinct $\mathrm{x}$-ray attenuation between the oil and water phases in the pore-scale images. The thermophysical properties of the two fluids are listed in Table I.

\section{B. Wettability alteration}

The surface wettability of the rock sample was altered to conditions representative of those in the subsurface, prior to conducting the experiment $[37,48]$. To alter the wettability, the rock was placed in contact with crude oil at conditions of high temperature and pressure, which allows for the adsorption of organic surface-active materials, from the crude oil, onto the rock surfaces, rendering them oil wet [43]. To achieve this, we performed the following steps. (i) First, the rock was cleaned using methanol and left to dry in an oven for $24 \mathrm{~h}$. (ii) The rock pore space was then fully saturated with formation brine (water containing the same salt composition as the aqueous phase in the reservoir from which the rock was extracted) at high temperature $\left(80^{\circ} \mathrm{C}\right)$ and high pressure $(10 \mathrm{MPa})$. (iii) Forty pore volumes of crude oil, from the same reservoir in the Middle East, were initially injected from the bottom of the sample with an increasing flow rate, from 0.001 to $0.1 \mathrm{ml} / \mathrm{min}$. The flow direction was then reversed and crude oil was injected from the top with the same flow rates and total volume. (iv) After this, five pore volumes of fresh crude oil were injected into the sample daily for a week at $0.05 \mathrm{ml} / \mathrm{min}$. (v) The sample was then maintained at the initially established high pressure and temperature $\left(80^{\circ} \mathrm{C}\right.$ and $\left.10 \mathrm{MPa}\right)$ conditions for another three weeks. (vi) Finally, the sample was removed and placed in a sealed crude oil bath at $80^{\circ} \mathrm{C}$ before it was transported to the synchrotron light source facility. Refer to Table S1 in [44] for the crude oil properties.

\section{Flow experiment and synchrotron imaging}

The experimental apparatus used to conduct the flow experiment in the synchrotron light source facility is shown in Fig. 1. The sample was removed from the crude oil bath and fitted in a cylindrical Viton sleeve. The top and bottom sides of the Viton sleeve were then attached to metal endpieces which were connected to the outlet and inlet flow lines, respectively. This configuration was assembled inside a Hassler-type flow cell made of carbon fiber that is X-ray transparent. The flow cell was then placed in front of the synchrotron beamline to start the experiment.

The flow experiment was initiated by flushing 20 pore volumes of the oil phase (doped $n$-decane) through the sample at a flow rate of $0.1 \mathrm{ml} / \mathrm{min}$ to replace the crude oil used to alter the wettability. This step is essential to avoid the formation of emulsions during the experiment [49]. The system was then pressurized to the experimental conditions $(8 \mathrm{MPa})$ and the confining pressure to $10 \mathrm{MPa}$. The temperature in the system was then raised to the experimental temperature $\left(60^{\circ} \mathrm{C}\right)$ using an Omega flexible heater. At this point, a single high-quality scan of the oil-saturated rock was acquired (hereafter called the reference oil scan). While oil initially occupied almost $99 \%$ of the macropore space before water injection (measured on the reference oil scan), water was initially present in the microporosity and in the corners of the pore space.

The dynamic experiment was then started by injecting water into the oil saturated rock at a very low flow rate, $0.15 \mu \mathrm{l} / \mathrm{min}$, achieving a capillary number $(\mathrm{Ca}=\mu q / \sigma$, where $\sigma$ is the oil-water interfacial tension, $\mu$ is the viscosity of water, and $q$ is the Darcy velocity of water) of $2.09 \times$ $10^{-9}$, which resulted in capillary-dominated flow conditions. Furthermore, the low flow rate enabled the flow dynamics 


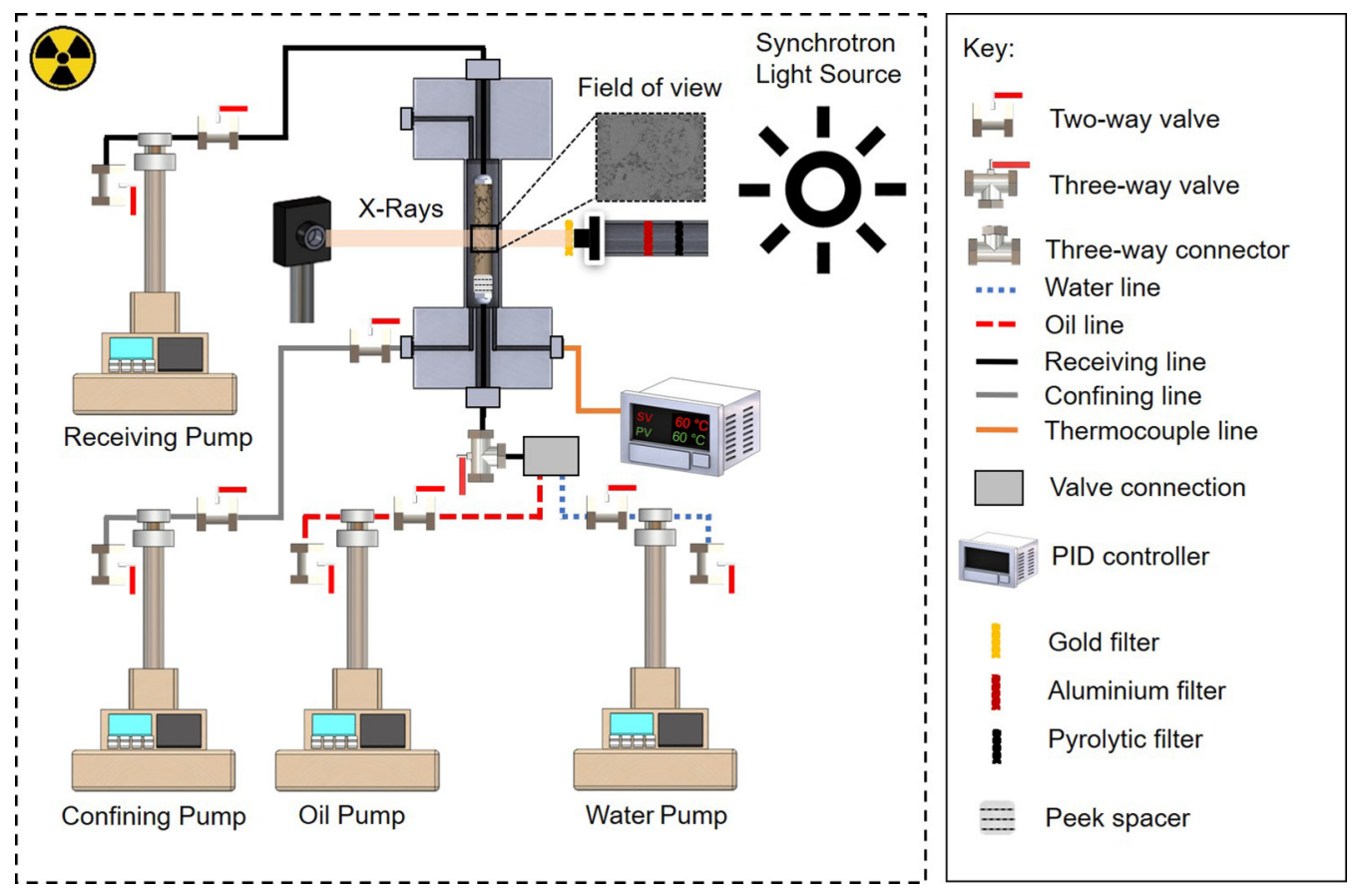

FIG. 1. High-pressure, high-temperature flow apparatus used to conduct the water injection experiment. The apparatus consisted of four syringe pumps, a carbon fiber flow cell, flexible heating jacket connected to a PID controller, a PCO edge camera, and a synchrotron light source.

to be captured with a temporal resolution of 70 s. During water injection, the center of the sample was continuously scanned (hereafter called dynamic water injection scans). No, or minimal, displacement dynamics were observed after 92.1 min of water injection, and hence the experiment was terminated.

The fast time-resolved synchrotron imaging was performed using a high photon flux pink beam with a peak photon energy of $15 \mathrm{keV}$. The $\mathrm{x}$ rays were filtered by placing a $1.3-\mathrm{mm}$ pyrolytic carbon filter, a 3.2-mm aluminum filter, and a $10-\mu \mathrm{m}$ gold filter in the beamline. Only the center of the sample was imaged during the experiment, giving a $4.5 \times 4.5 \times 3.8 \mathrm{~mm}^{3}$ field of view with a PV of $0.02 \mathrm{ml}$ as shown in Fig. 1. The imaged field of view covered the whole cross-sectional area of the sample. Imaging started when water was detected in the peek spacer. The size of the images was $1280 \times 1280 \times$ 1080 voxels, with a voxel size of $3.5 \mu \mathrm{m}$. The high-quality oil scan was acquired with a total of 2000 projections and 0.15 -s exposure time. During water injection a total of 76 tomograms were acquired over a period of $92.1 \mathrm{~min}$, with 700 projections and 0.065-s exposure time. The lower number of projections and exposure time allowed for the dynamic images to be acquired with a high temporal resolution (a complete tomogram was acquired every $70 \mathrm{~s}$ ).

\section{Image processing}

The tomograms were then reconstructed to build threedimensional (3D) pore-scale images of the rock and fluids within it. Figure 2 shows the same two-dimensional slice of the 3D pore-scale images taken after oil injection [Fig. 2(a)] and at the end of water injection [Fig. 2(b)]. All images were segmented using the machine-learning-based trainable Weka segmentation method [50,51]. No filtering was applied to the images prior to segmentation as it can have an adverse effect on the quality of Weka segmentation [43]. Two approaches were adopted to segment the reference oil scan and the water injection scans. In the case of the high-quality oil scan, the classifier was trained by manually selecting voxels that belong to the oil and rock phases, which was then applied to segment the whole image [see Fig. 2(d)].

The segmentation of the dynamic water injection scans was performed in four steps. (i) First, all the 76 time-series raw water injection images were registered to the raw oil reference scan. (ii) Each water injection scan containing three phases (water, oil, and rock) was then subtracted from the oil reference scan, which contains mainly two phases (oil and rock), creating a subtracted image where the water phase can be clearly distinguished [see Fig. 2(c)]. (iii) Water was then segmented using Weka by training the classifier to identify the voxels that belong to the water phase [see Fig. 2(f)]. (iv) The segmented water phase was then masked on the segmented oil reference scan resulting in the final segmented water injection image [see Fig. 2(e)]. A cylindrical mask was then applied on the segmented images to remove the unwanted regions, e.g., the Viton sleeve. The fast random algorithm was employed during all Weka segmentations alongside the mean and variance texture filters.

\section{E. Wettability characterization methods}

The segmented 3D pore-scale images can be used to characterize the wettability of the rock surface directly in situ. Wettability can be inferred from the measured spatial distribution of contact angles between the fluids within the pore space, also known as the geometric contact angle $\theta_{g}$ [52-56]. While 

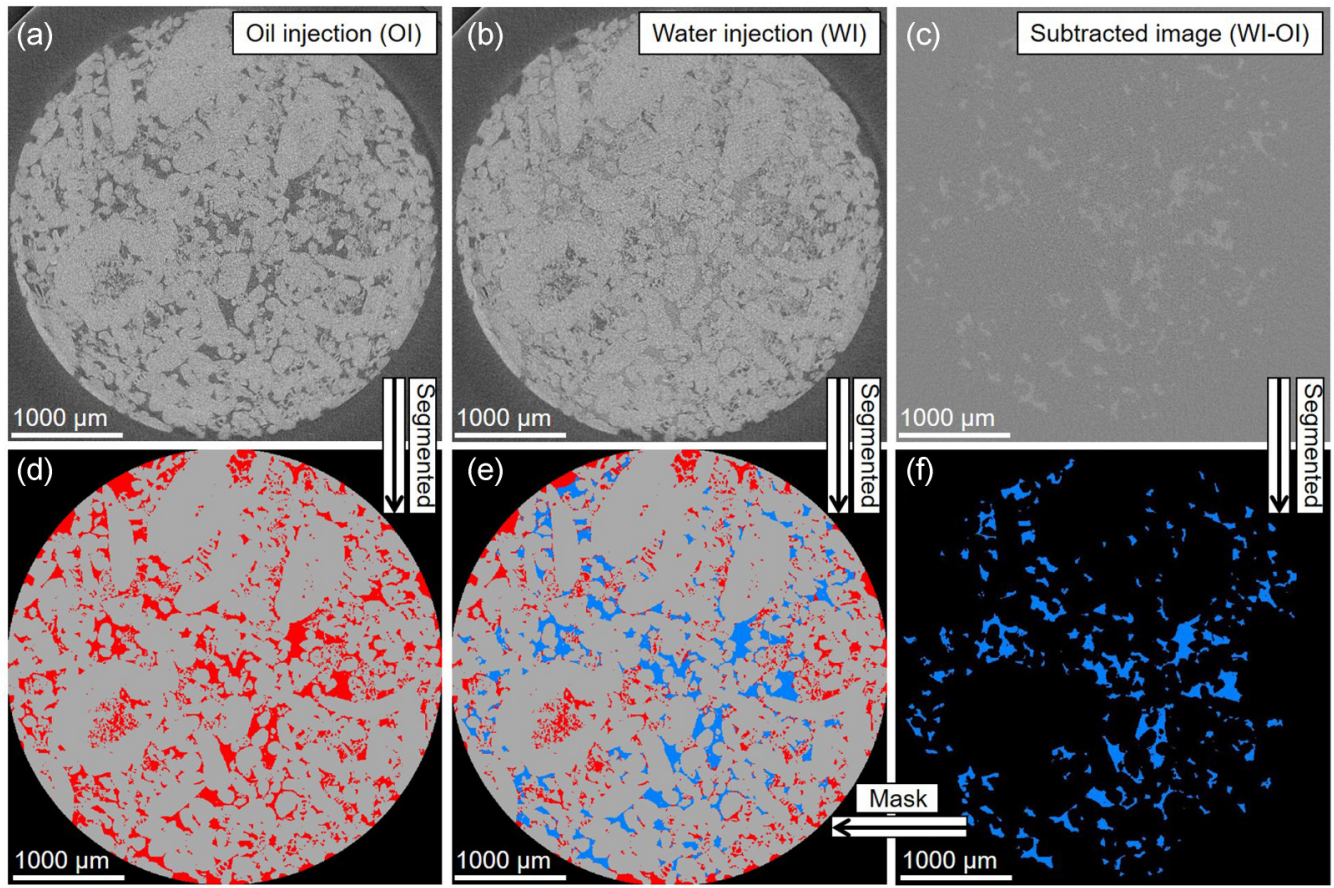

FIG. 2. Image segmentation workflow. The raw reference oil scan, $3.5 \mu \mathrm{m}$ voxel size, in (a) was segmented directly using weka segmentation [see (d)]. To segment the water injection scan, $3.5 \mu \mathrm{m}$ voxel size, in (b), it was first subtracted from the reference oil scan, in (a), to clearly distinguish the water phase, as shown in light gray in (c). The water phase in (c) was then segmented using weka, as shown in (f), and then masked on the segmented oil scan in (d) to give the final segmentation of the water injection scan [see (e)]. The mean and variance texture filters were used during weka segmentation.

the geometric contact angle provides useful information on wettability on a pore-by-pore basis, its value is measured at rest and hence it does not necessarily represent the actual value encountered during displacement because of contact angle hysteresis [57]. Moreover, it has been shown that the use of spatially distributed geometric contact angle values were insufficient to reproduce the water flooding behavior in altered-wettability media [58].

Therefore, we also use an alternative approach to define the contact angle associated with the fluid displacement in our system, known as the thermodynamic contact angle $\theta_{t}$. Here $\theta_{t}$ is computed from an energy balance and considers the changes in saturation and interfacial areas from two consecutive water injection images (time steps) [59]

$$
\cos \theta_{t}=\frac{\kappa \phi \Delta S_{w}+\Delta a_{w o}}{\Delta a_{w s}},
$$

where $\Delta S_{w}, \Delta a_{w o}$, and $\Delta a_{w s}$ are the differences, between two time steps, in water saturation, and specific interfacial areas between water and oil, and water and solid measured on the macroporosity. In addition, $\kappa$ is the total curvature of the oil-water interface, discussed below, and $\phi$ is the imagedbased macroporosity. In our dynamic water injection images, we compute the thermodynamic contact angle between all the time steps and compare its value to the distribution of geometric contact angle.

\section{F. Pore-filling analysis method}

To quantitatively assess the order of pore filling during water injection, we developed an in-house algorithm that detects the size of the filled elements as the water front progresses through the pore space. For each time step $i$, the algorithm identifies the pores occupied by the advancing water front and measures the size of the throats connected to it, also known as available throats for invasion, using the maximal ball network extraction code [60,61]. A throat is considered available whenever two pores connected to it are occupied by different phases (oil and water). Subsequently, the algorithm records the inscribed radius of the available throats, in $i$, that become invaded by water in the next time step $i+1$. An available throat is considered invaded when both pores connected to it are filled with water and its center is also occupied by water. Finally, the algorithm generates a plot showing the size of the invaded available throats at time step $i+1$ against the size of the available throats at time $i$ to illustrate the order of filling during water injection. This approach is different from a conventional occupancy analysis that depicts the size of the invaded pores or throats at each time step against the size of all the pores or throats in the rock. Here we only consider the throats available for invasion, which are adjacent to the advancing water front.

If the rock has become uniformly oil wet (water repellent), we expect water injection to be a drainage process. If displacement proceeds by invasion percolation then we expect at every time step the largest available throats to be filled [14].

\section{G. Minkowski functionals}

In addition to characterizing the in situ wettability and order of pore filling, the segmented pore-scale images can be used to compute the four Minkowski functionals, which are 

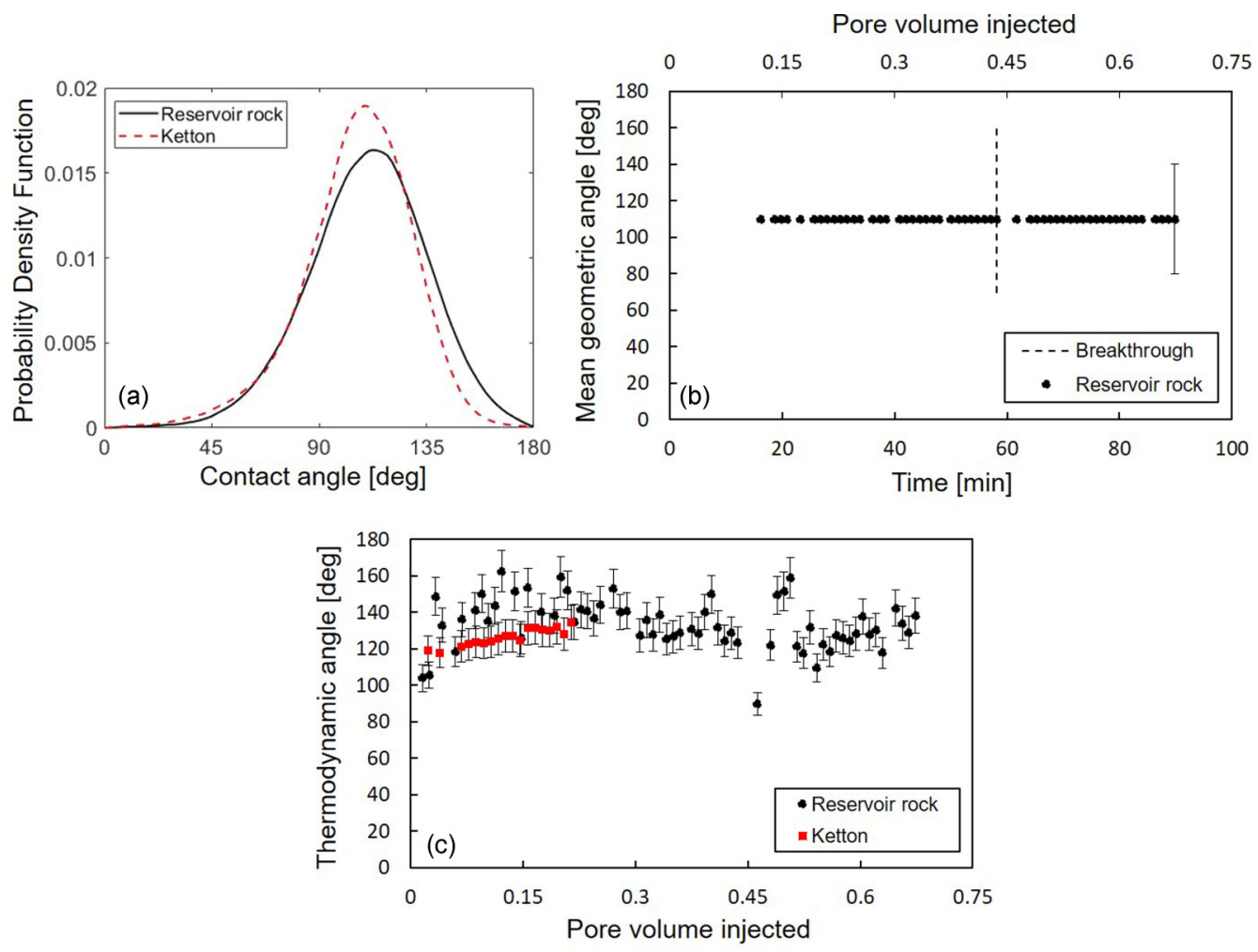

FIG. 3. (a) Normalized histograms of the measured in situ contact angles between oil and water in the reservoir sample (black) and in the Ketton limestone sample (red) at the end of water flooding. (b) Mean of the calculated geometric contact angle distributions in the reservoir sample plotted as a function of time and pore volume injected. (c) Calculated average value of the thermodynamic contact angle, using Eq. (2), with pore volumes injected in the reservoir sample (black) and in the Ketton sample (red). Error bars in (b) show the standard deviation, while in (c) it indicates the uncertainty in the measurements. The geometric and thermodynamic contact angles measured on Ketton are from Scanziani et al. [36].

morphological measures that provide a complete geometrical characterization of the fluids within the pore space [62-64]. The zeroth-order Minkowski functional $M_{0}$ is the volume. In two-phase flow, this can be defined as the volume of each phase, which we obtained to calculate the saturation of the oil and water phases

$$
S=\frac{v}{\phi V}
$$

where $S$ is the fluid phase saturation defined here only in the macroporosity, $v$ is the volume of the fluid phase in the pore space, $\phi$ is the macroporosity, and $V$ is the image size (total volume).

Interfacial area is the first-order Minkowski functional $M_{1}$, from which we can define the specific interfacial area $a$ between the fluids and the fluids and the solid ( $a$ has units of 1/length) [65]:

$$
a=\frac{M_{1}}{V} .
$$

We computed the specific interfacial area between water and oil $\left(a_{w o}\right)$, oil and solid $\left(a_{o s}\right)$, and water and solid $\left(a_{w s}\right)$ from the segmented images.

The second-order Minkowski functional $M_{2}$ is the total curvature (the sum of the two principal curvatures) of the interface between two phases $\kappa$. Here $\kappa$ can be related to the capillary pressure $P_{c}$ through the interfacial tension $\sigma$ using the Young-Laplace equation [66]

$$
P_{c}=\sigma \kappa
$$

We estimated the capillary pressure between oil and water by measuring the curvature of their interface on the pore-scale images using a method previously described in the literature [66-71]. The 3D oil-water interface was extracted from the image and then smoothed with a kernel size of 5 in the direction of the aqueous phase (water). The average curvature was measured on the smoothed surface and multiplied by the interfacial tension (Table I) to obtain the capillary pressure from Eq. (5).

The third, and last, Minkowski functional $M_{3}$ is the surface integral of the Gaussian curvature, or the product of the two principal curvatures $\kappa_{1}$ and $\kappa_{2}$ [64]. The Gaussian curvature of the fluid-fluid interface can be used as a measure of the connectedness of the fluid phases in the pore space.

\section{RESULTS AND DISCUSSION}

In this section, we analyze the segmented images to investigate the pore-scale dynamics during water injection in the reservoir rock. First, in Sec. III A, we compute the geometric and thermodynamic contact angles for each time step to characterize the wettability of the rock; we suggest that the thermodynamic angles provide a better quantification of the wettability during displacement. In Sec. III B, we examine 


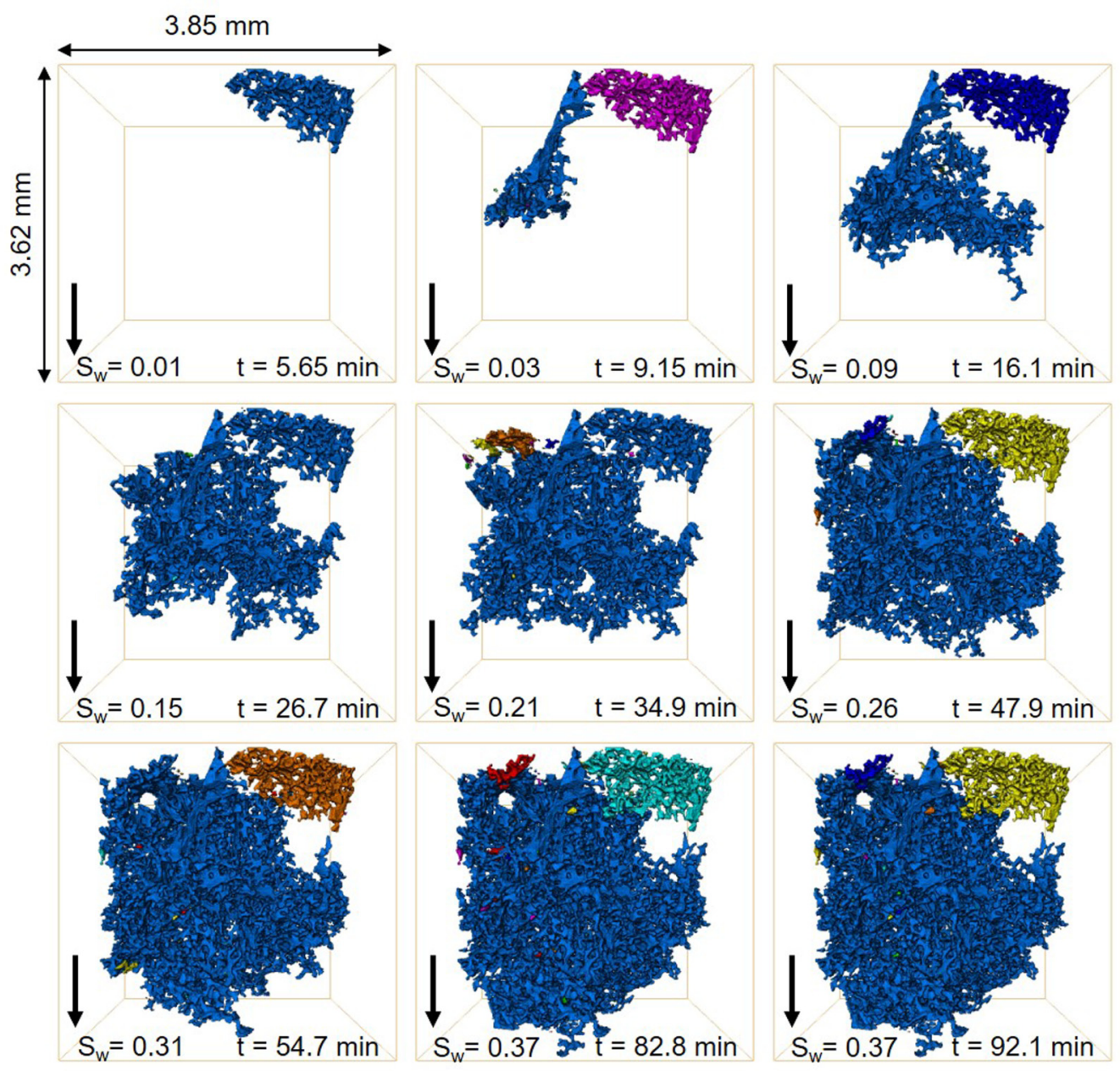

FIG. 4. Three-dimensional maps of the water phase connectivity during water injection shown at different time steps. During invasion, water advanced as a connected front displacing oil in the pore space. This displacement was accompanied by apparent drainage dynamic events, including Haines jumps $(t=9.15 \mathrm{~min})$ and Roof snap-off $(t=16.1,47.9,54.7,82.8$, and $92.1 \mathrm{~min})$. The black arrow points towards the direction of flow.

the pore-filling events by qualitatively and quantitatively assessing (1) the invasion patterns, (2) Haines jumps, and (3) snap-off events. We demonstrate that the water advance is an invasion percolation process. Finally, in Sec. III C, for each water injection time step, we quantify the Minkowski functionals describing (1) fluid saturations, (2) specific interfacial areas between the fluids and the fluids and rock, (3) oil-water capillary pressure, and (4) Gaussian curvatures.

\section{A. Geometric and thermodynamic contact angles}

The distribution of in situ geometric contact angles $\theta_{g}$ between oil and water was measured in a subvolume of size $1.75 \times 1.75 \times 1.75 \mathrm{~mm}^{3}$, located in the center of the field of view, during water injection using the automated method developed by AlRatrout et al. [53]. Figure 3 shows the distribution of the geometric oil-water contact angles in our reservoir rock compared to that measured on Ketton limestone by Scanziani et al. [36]. The mean of the geometric contact angle distribution in the reservoir rock is $110^{\circ}$ with a standard deviation of $\pm 20^{\circ}$. This indicates that the rock surfaces are predominately oil-wet such that the rock tends to be in contact with oil; water, the nonwetting phase, resides in the centers of the pores surrounded by oil-wetting layers (see Figs. 4 and 5). Given the oil-wet nature of the rock, we expect the displacement of oil by water to be drainagelike. Moreover, the wide range of pore sizes in the heterogeneous reservoir sample (Fig. S1 in [44]) suggests that displacement is more likely to be controlled by radius than contact angle, which is discussed further in Sec. III B 1. The average geometric contact angle distribution in the reservoir rock is similar to that obtained on a Ketton limestone sample, $109^{\circ} \pm 19^{\circ}$, where the same wettability-alteration protocol was employed [36] [Fig. 3(a)]. However, as we will show later, the displacement behavior is very different. Furthermore, we observe that the mean geometric contact angle remains constant throughout the water flooding experiment in the reservoir rock [see Fig. 3(b)].

Nevertheless, as mentioned earlier, the geometric contact angle is measured on a fixed oil-water interface. Many of the contacts between oil, water, and the solid are pinned and have a hinging contact angle between the low water-wet value when oil first entered the rock and a higher value needed for water to advance across an altered-wettability surface. Hence, the 


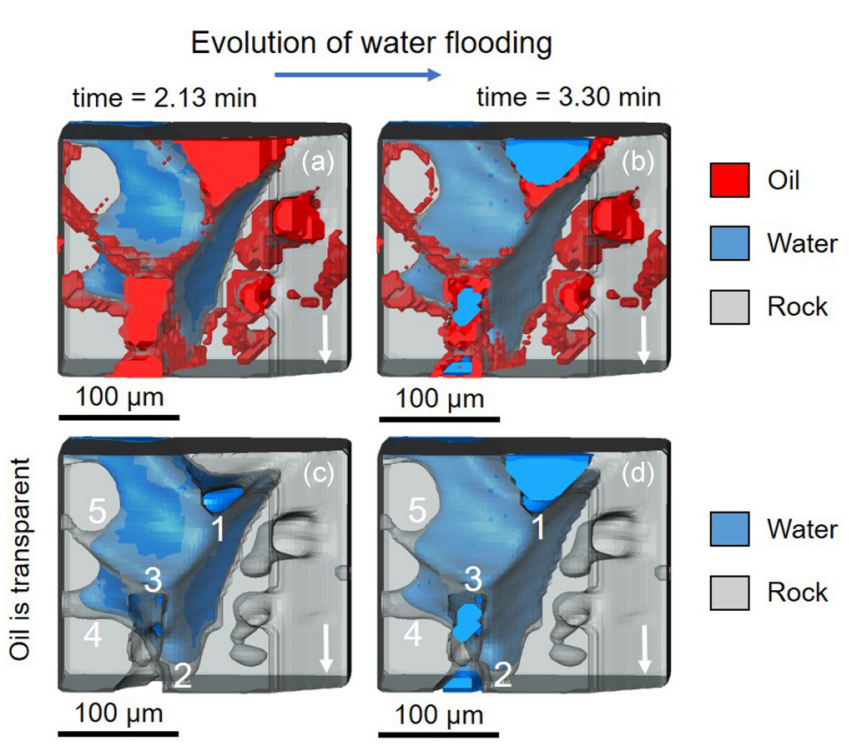

FIG. 5. Three-dimensional pore-scale images of a single pore $\left(210 \times 123 \times 175 \mu \mathrm{m}^{3}\right)$ showing the invasion pattern of water. (a) At time $=2.13 \mathrm{~min}$, there are five throats connected to the pore that are available for subsequent water invasion. These throats are labeled from 1 to 5 in decreasing order of size in (c), where oil was rendered transparent to make it possible to visualize the throats. (b) and (d) At time $=3.30 \mathrm{~min}$, the largest throats $1-3$ get invaded by water, which indicates that water injection in an oil-wet medium is an invasion percolation process. The white arrow points towards the direction of flow, while the blue arrow refers to the evolution of water invasion.

geometric values do not necessarily represent the oil-water contact angles encountered during displacement, with a tendency to underestimate the values [57,59]. We therefore used Eq. (2) to calculate a thermodynamically consistent contact angle $\theta_{t}$ for each time step. The calculated thermodynamic contact angles are shown in Fig. 3(c): this represents the average oil-water contact angle for displacement consistent with the change in surface energy estimated from the images. Figure 3(c) indicates that the thermodynamic contact angle in the reservoir rock has a stable trend during water injection, with an average value of approximately $135^{\circ} \pm 10^{\circ}$. A lower $\theta_{t}$ was observed early on in the displacement, $103^{\circ} \pm 10^{\circ}$ and $105^{\circ} \pm 10^{\circ}$, indicating that, initially, water preferentially fills the less oil-wet pores, followed by the filling of increasingly oil-wet regions of the pore space. In the previous study on Ketton, the thermodynamic angle increased from $110^{\circ}$ to $130^{\circ}$, shown in red in Fig. 3(c), throughout the displacement; however, it was still lower than the thermodynamic angle for the reservoir rock [36]. Hence, we observe that the thermodynamic angle provides a better discrimination between the two cases than the geometric angle which displayed a similar distribution. Although the difference in wettability captured by the thermodynamic angle is relatively modest, combined with a more heterogeneous pore-size distribution, it leads to a distinct displacement behavior, as presented next.

Furthermore, a recent modeling study has shown that to match experiments of water flooding in rocks with altered wettability, it is insufficient to use the geometric contact angle; instead, a larger contact angle should be used to account for contact angle hysteresis in the more oil-wet regions [58]. This implies that the thermodynamic contact angle better captures the displacement in a model.

\section{B. Pore-filling events}

The pore-scale dynamics of water flooding were imaged for a total of $92.1 \mathrm{~min}$, after which there was no significant change in the oil and water configurations in the pore space. Water breakthrough in the imaged field of view, $3.62 \mathrm{~mm}$ in length, occurred after $58.2 \mathrm{~min}$, which corresponds to an injection of $0.45 \mathrm{PV}$ of water. Figure 4 and movie $\mathrm{S} 1$ in [44] show images of the advancing water phase acquired at different time steps; each color represents a different water cluster.

We observe that water advances as a connected front displacing the oil phase in the pore space, which indicates that water injection in our oil-wet reservoir rock is a drainage process. Layer flow was inferred during displacement as oil, the wetting phase, was observed in the corners and roughness of the pore space (see Fig. S2 and movie S2 in [44]) as well as in the pore centers. Furthermore, water invasion was accompanied by drainage dynamics associated with interface advancing and retraction, e.g., Haines jumps and Roof snapoff (see Fig. 4); this further confirms that drainage was the displacement process in the experiment. Drainage and its associated dynamics were previously imaged in water-wet systems [18,34] but not in oil-wet media. Figure 4 shows that drainage dynamics continued even after breakthrough as water displaced more oil out of the pore space. This contrasts with the experiment on Ketton limestone, where no displacement was observed after breakthrough [36].

\section{Invasion percolation}

The time-series segmented images were analyzed to characterize the pore-filling order during water injection. First, we qualitatively assess the order of filling in a single pore occupied by oil and water (see Fig. 5). Figure 5(a) illustrates that water resides in the centers of the pore space confining oil to wetting layers and small corners, which is a common characteristic of predominantly oil-wet media [48]. At time equal to $2.13 \mathrm{~min}$ [Fig. 5(a)] there are five available throats for water to pass through to progress to the next pores. These available throats are labeled from 1 to 5 in a decreasing order of size in Fig. 5(c), where the oil phase was rendered transparent to make it possible to visualize the available throats. At time equal to 3.30 min [Figs. 5(b) and 5(d)], we observe that water invaded the centers of the largest available throats (1-3) to pass through, as they required the lowest absolute capillary entry pressures [Eq. (1)]. We presume that as water pressure increases during water injection, water first had sufficient pressure to invade the largest throat (1) and then progressively filled the smaller throats (2 and 3 ). However, this happens on a much shorter timescale than that required for a single scan, and hence was not captured in the experiment. Overall, the throats were filled in order of size, with the largest filled first, which indicates that water injection in this rock is an invasion percolation process.

Using the pore-filling analysis described in Sec. IIF, the filling sequence was quantified in all the pores adjacent to the advancing water front in the dynamic images during water 

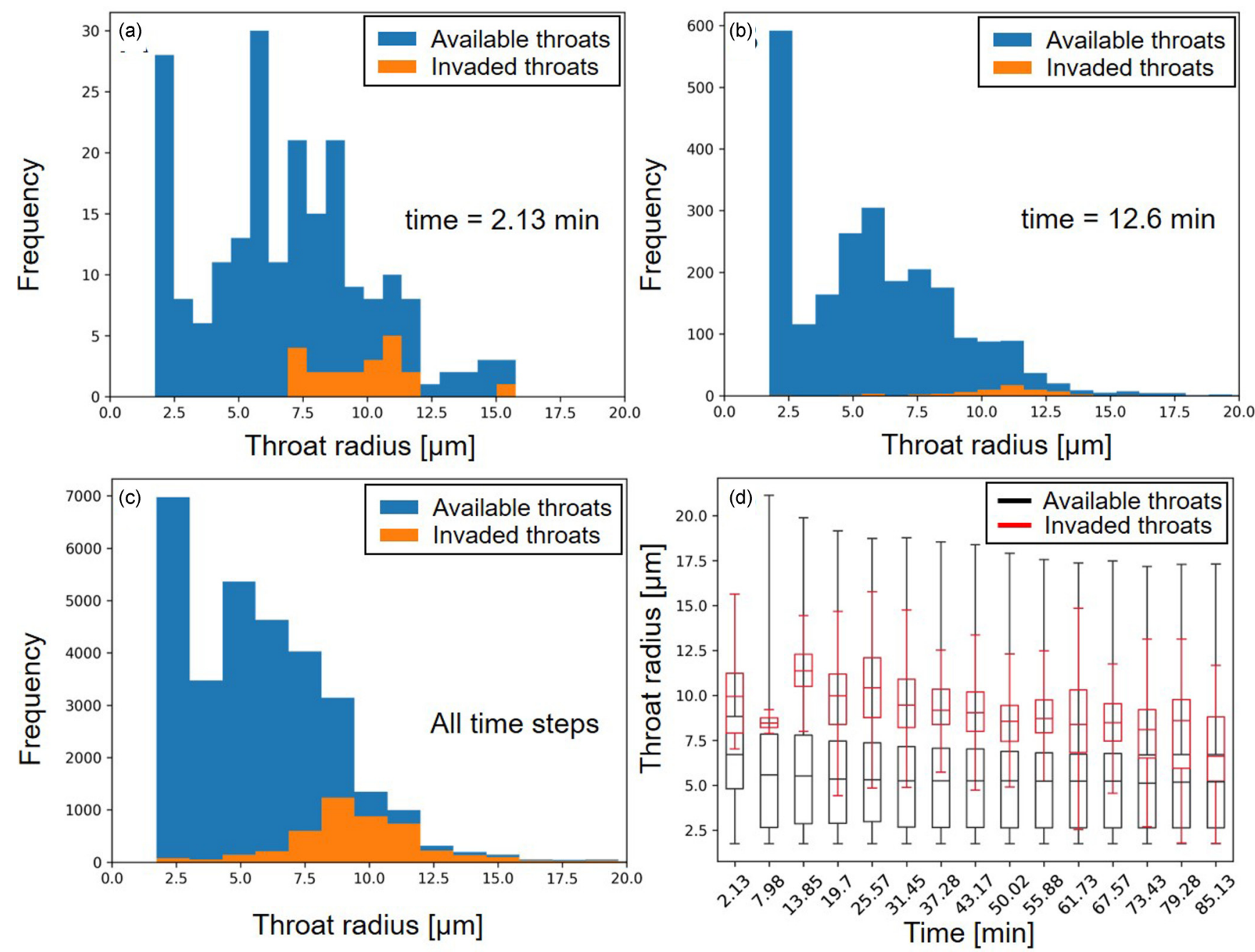

FIG. 6. Pore-filling analysis conducted by plotting the size of invaded throats against the size of all the available throats for water invasion. The sizes of throats filled are plotted at time (a) $2.13 \mathrm{~min}$ and (b) $12.6 \mathrm{~min}$. (c) Accumulation of the pore-filling analysis results at all the time steps. (d) Box plot of the cumulative pore-filling results in (c) shown as a function of time.

injection. For each time step, the size of the invaded throats is plotted against all the available throats for invasion (see Fig. 6). Figures 6(a) and 6(b) show that the advancing water front invades the largest available throats at time steps of 2.13 and $12.6 \mathrm{~min}$, respectively, which again confirms that water injection in oil-wet media is an invasion percolation process. This behavior is further inferred from the cumulative result of all the 76 time steps shown in Fig. 6(c), indicating that water almost always invades the largest throats during displacement.

To show the significance of invasion percolation in our oil-wet rock, we generated a box plot for the invaded throats against the available throats for every five time steps [see Fig. 6(d)]. Figure 6(d) shows that the portion of the available throats invaded by water always lies within the largest $5 \%$ of the available throats for invasion. This indicates that the displacement in our heterogeneous oil-wet system is mainly size controlled, as filling larger throats requires a lower absolute value of the oil-water capillary pressure, and that wettability has a little effect. This is attributed to the wide pore size distribution of the rock sample selected (Fig. S1 in [44]). The impact of wettability on displacement is marked early on, where the less-oil-wet pores were filled by water first (see
Fig. 3). It is also unlikely that the later filling sequence is determined by a correlation between contact angle and throat radius, where the larger throats also have a smaller contact angle (favoring filling): Previous work on a similar reservoir rock has shown that there is weak tendency for larger pores and throats to be more oil-wet with large contact angles [9].

Wettability is expected to play a greater role in displacement in systems with a more uniform pore and throat size distributions. More specifically, it has been previously observed in the Ketton limestone sample with similar wettability conditions but a narrower pore size distribution, where both geometry and wettability controlled the displacement sequence and pores of all size were filled during water injection [36].

\section{Haines jumps}

Haines jumps were observed on single- and multiple-pore levels during water injection. Figure 7 shows the rapid filling of multiple pores during a Haines jump and quantifies the specific interfacial area of water before and after its retraction from the narrower regions. First, we notice a slow increase in the water saturation between time steps 3.30 and $7.98 \mathrm{~min}$ 


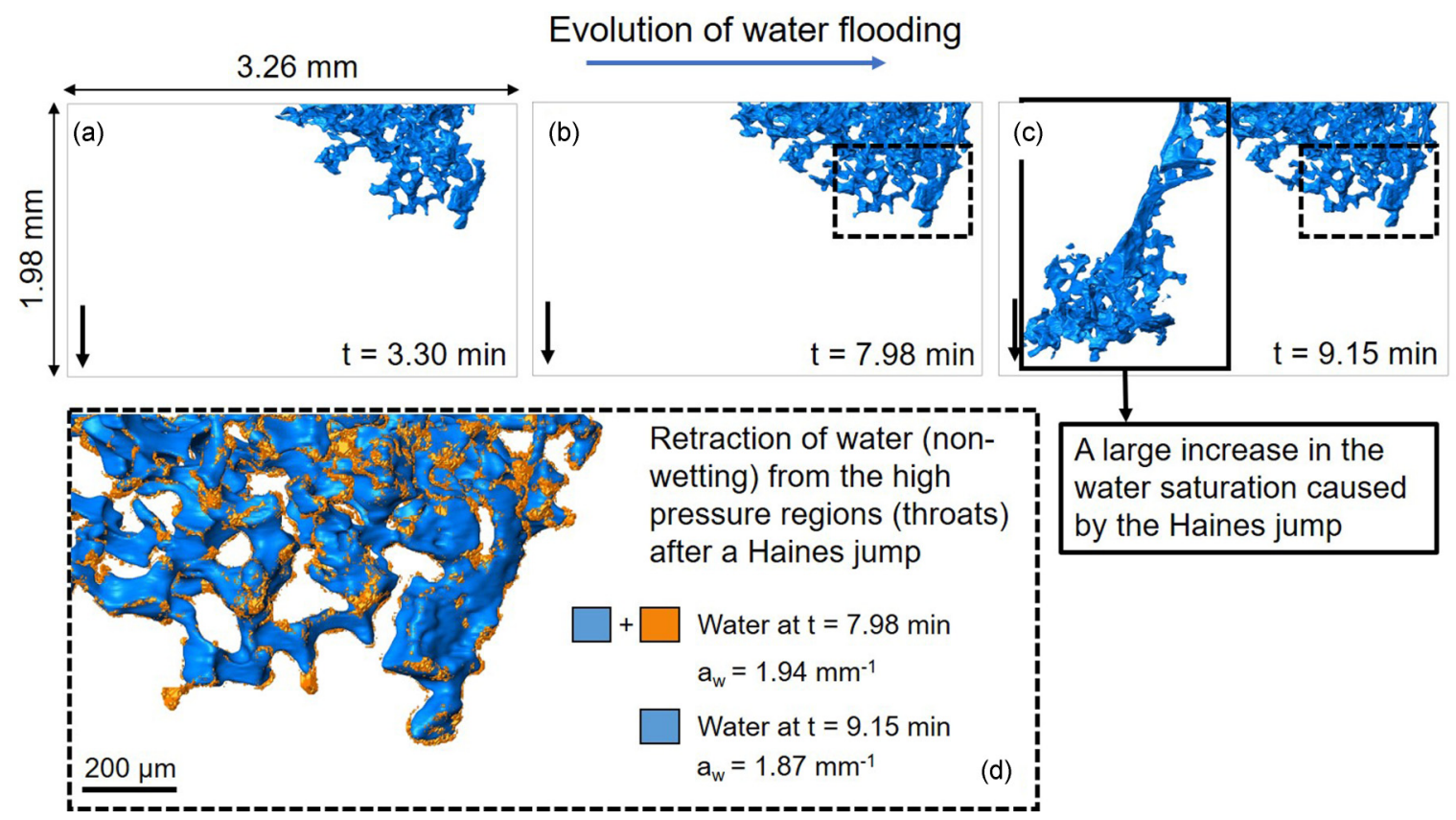

FIG. 7. Three-dimensional images of the water phase at different time steps illustrating the rapid filling of multiple pores during a Haines jump. (a) and (b) Slow increase in the water saturation, which was followed by a large increase in the water saturation caused by the Haines jump in (c). (d) The specific interfacial area of water is lower in the high-pressure region, marked by the dashed line, after the Haines jump due to water retraction. The black arrow points towards the direction of flow, while the blue arrow refers to the evolution of water invasion.

[Figs. 7(a) and 7(b), respectively]. However, as soon as the Haines jump occurs at time equal to $9.15 \mathrm{~min}$, water rapidly fills multiple pores, resulting in a large increase in the water saturation [see Fig. 7(c)]. The movement of the advancing water interface during a Haines jump is very rapid and cannot be captured with the temporal resolution of this experiment (70 s); micromodel studies have shown that the pores drain on a millisecond timescale [72].

Furthermore, we observe a rearrangement of the water phase in the pore space associated with the Haines jump,

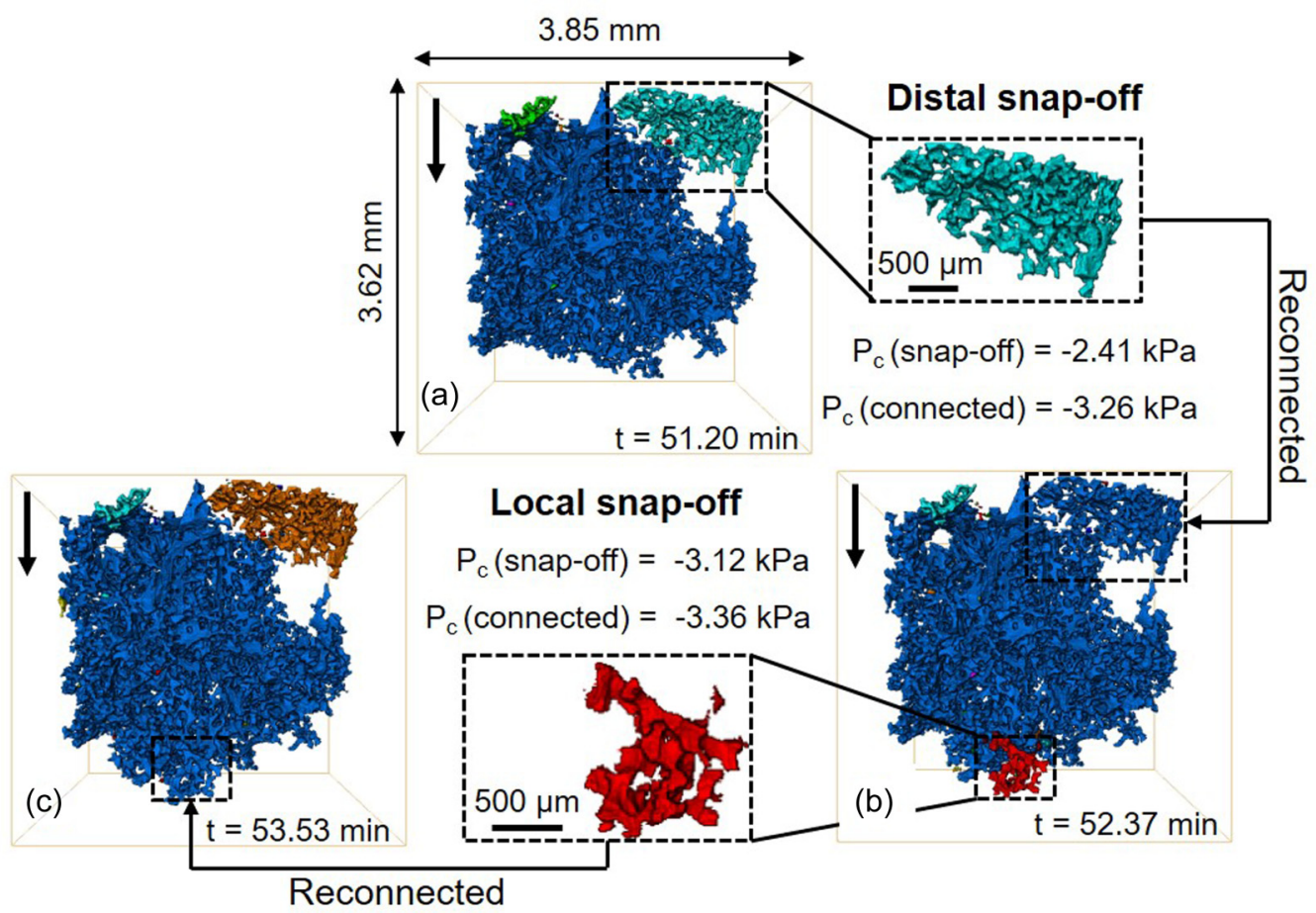

FIG. 8. Three-dimensional images illustrating the occurrence of local and distal snap-off during water injection. Only the water phase is shown; each color represents a disconnected water phase cluster. The black arrow points towards the direction of flow. 

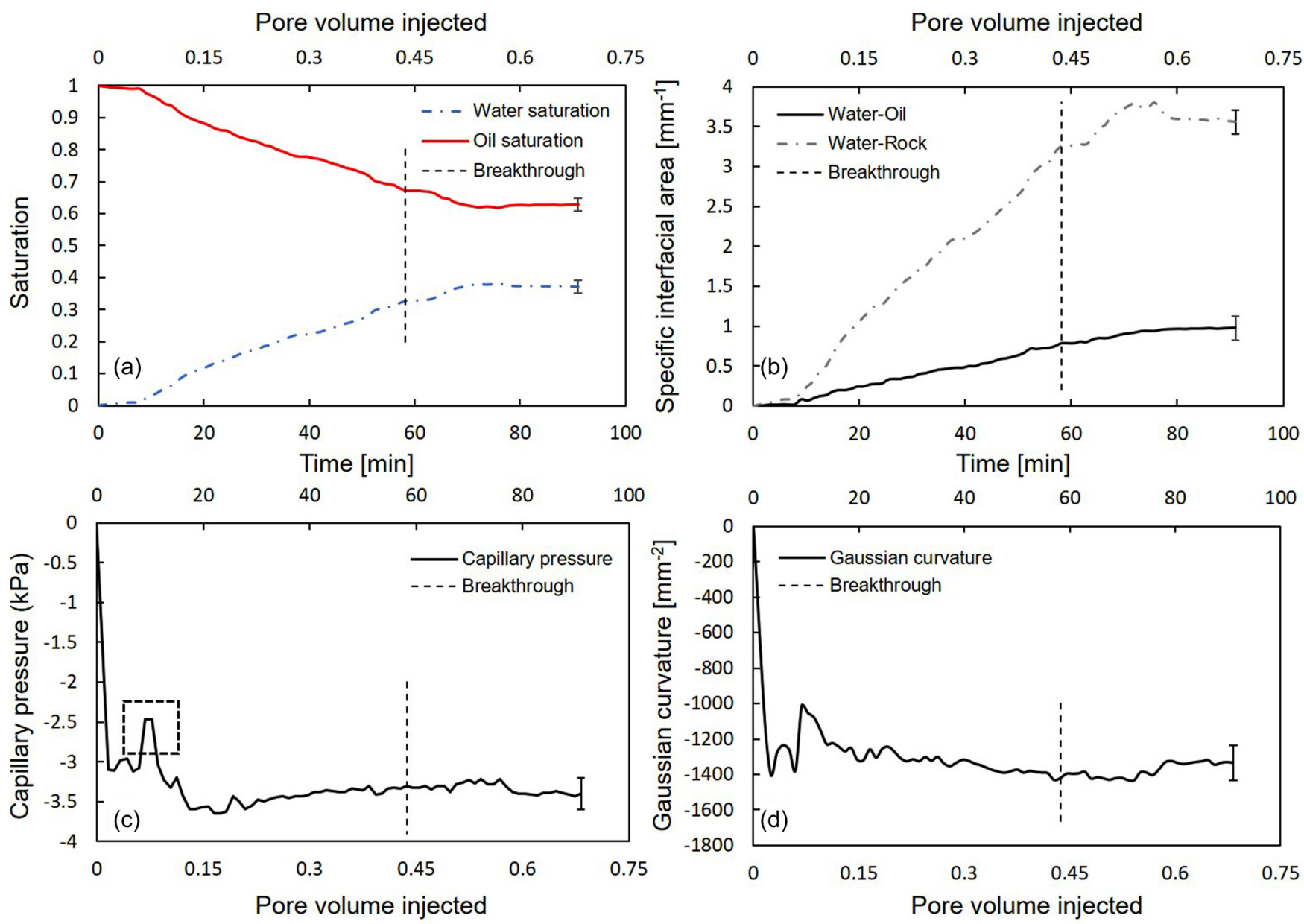

FIG. 9. Minkowski functionals. (a) Oil and water saturation profiles as a function of time and pore volume injected. (b) Change in specific interfacial areas of oil-water and water-rock interfaces with time and pore volume injected. (c) Oil-water capillary pressure plotted as a function of time and pore volume injected. (d) Change in Gaussian curvature of the oil-water interface with time and pore volume injected. The dashed line represents the time of water breakthrough in the imaged field of view. The dashed square in (c) shows a sudden change in the local capillary pressure which corresponds to the filling of multiple pores during a Haines jump, illustrated in Fig. 7. Error bars indicate the uncertainty in the measurements.

where water retracts from the high-pressure regions (throats) and flows towards regions of lower water pressure to supply the rapid filling. This is shown in Fig. 7(d), where water has a lower specific interfacial area of $1.89 \mathrm{~mm}^{-1}$ at time equal to $9.15 \mathrm{~min}$ after the Haines jump compared to $7.98 \mathrm{~min}$, where water had a specific interfacial area of $1.94 \mathrm{~mm}^{-1}$. We notice that water, shown in blue, has retracted from the throats, shown in orange, in the region marked with a dashed line. The effect of multiple-pore filling during Haines jumps is more marked early in the water flooding experiment, when most pores are occupied by oil only.

\section{Local and distal snap-off}

We observed the two types of snap-off, local and distal, during the water flooding experiment (see Fig. 8). Distal snap-off occurs due to the retraction of water, the nonwetting phase, from the narrower regions of the pore space during a Haines jump (Fig. 7), some distance away from the jump itself. The retraction of water is an imbibition process, which can result in snap-off and disconnection of the water phase as shown in Fig. 8(a). Next we measured the capillary pressure in the system using the method described in Sec. II G. The measurements show a lower absolute value of the local capillary pressure for the disconnected water ganglion $(-2.41 \mathrm{kPa})$ compared to the connected water cluster $(-3.26 \mathrm{kPa})$, which indicates that the trapped water ganglion reaches a new state of capillary equilibrium in the pore space. Furthermore, as water invasion proceeds, its local capillary pressure decreases (higher water pressure), allowing it to reaccess the throat where snap-off occurred, and hence it will reconnect with the stranded ganglion as shown in Fig. 8(b).

Local snap-off occurs in the newly filled pores by the invading water front as shown in Fig. 8(b). As water passes through a narrow throat into the adjoining pore, the local capillary pressure will suddenly change, allowing the oilwetting layers in the throat to swell. If the capillary pressure reaches the threshold for snap-off, the throat will spontaneously fill with oil, trapping water as a disconnected ganglion in the center of the pore [73]. Again, the snapped-off ganglion attains a new position of equilibrium with a lower absolute value of capillary pressure $(-3.12 \mathrm{kPa})$ compared to 

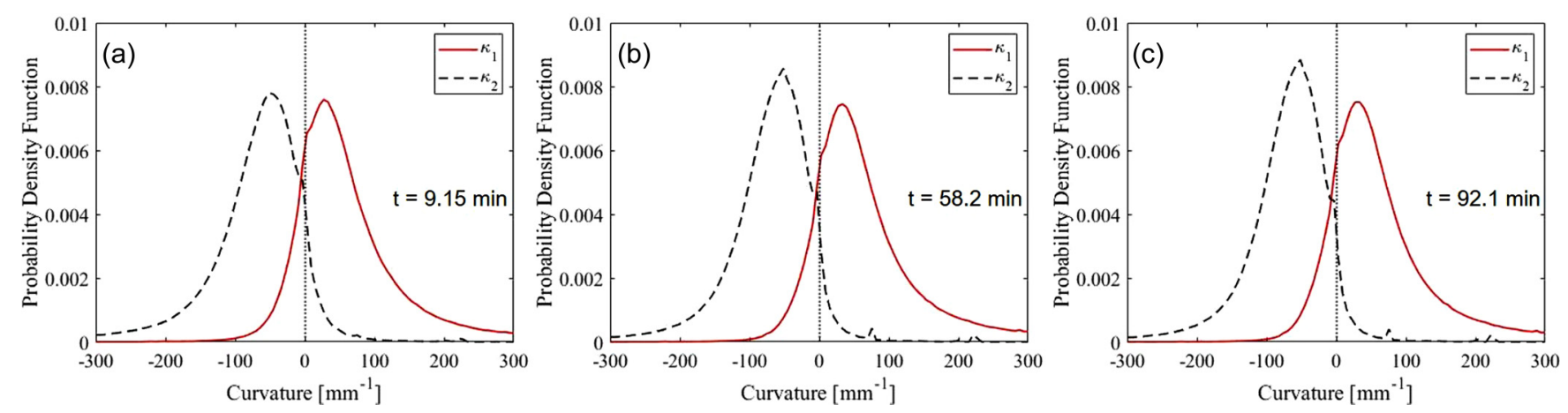

FIG. 10. Normalized histograms of the two principal curvatures $\kappa_{1}$ and $\kappa_{2}$ of the oil-water interface at three time steps: (a) $t=9.15$ min, (b) $t=58.2 \mathrm{~min}$, and (c) $t=92.1 \mathrm{~min}$. Here $\kappa_{1}$ is defined to be the larger curvature.

the connected water phase $(-3.36 \mathrm{kPa})$. As water progresses through the pore space, the trapped ganglion is reconnected [see Fig. 8(c)]. These snap-off events have been previously observed using fast synchrotron studies in water-wet media during oil invasion but not in oil-wet systems $[18,19]$.

\section{Saturation, interfacial area, and capillary pressure}

The saturation of oil and water in the macropores was computed using Eq. (3) on the segmented water injection images [see Fig. 9(a)]. Figure 9(a) and movie S3 in [44] show that the water saturation linearly increased with time even after the observed breakthrough in the imaged field of view (58.2 min). A noticeable increase in the water saturation was recorded at $9.15 \mathrm{~min}$, corresponding to the rapid filling of multiple pores caused by the Haines jump event shown in Fig. 7. The water and oil saturations stabilize to $(38 \pm 2) \%$ and $(62 \pm 2) \%$, respectively, after $78.1 \mathrm{~min}$, which corresponds to an injection of $0.58 \mathrm{PV}$ of water; further injection of water does not displace oil out of the pore space. The low oil recovery factor seen is attributed to water invading the centers of the pores, leaving oil connected in thick wetting layers in the corners and crevices of the pore space. This behavior was observed in previous water flooding static experiments conducted on the same rock type [74].

The oil-water and water-rock interfaces were extracted and smoothed [75] to measure their specific interfacial areas [Eq. (4)]. Figure 9(b) shows that the water-rock and oilwater specific interfacial areas increase with time during water injection.

Furthermore, the interfacial curvature of the extracted oilwater interface was measured and substituted in Eq. (5) alongside the interfacial tension, $52.1 \mathrm{mN} / \mathrm{m}$ (Table I), to characterize the capillary pressure of the system [see Fig. 9(c)]. A strongly negative capillary pressure, $-3.5 \mathrm{kPa}$, indicates that the macropores are indeed oil wet such that on average water bulges into oil with a higher pressure. This capillary pressure is higher than the value of $-2.4 \mathrm{kPa}$ measured for a Ketton sample which, as previously discussed, displayed more mixed-wet behavior [36]. The dashed square in Fig. 9(c) at 9.15 min shows the sudden change in the local capillary pressure that caused the Haines jump in Fig. 7.

The Gaussian curvature of the oil-water interface was computed and plotted as a function of time and pore volumes of water injected in Fig. 9(d). While the sum of the curvatures is negative, in most cases one curvature is negative and the other positive, giving a negative Gaussian curvature. This indicates that the phases are well connected in the pore space, implying that oil and water flow simultaneously, although oil flow is slow since it is confined to movement in wetting layers. Furthermore, we plotted, at three time steps $(9.15,58.2$, and $92.1 \mathrm{~min})$, the two principal curvatures $\left(\kappa_{1}\right.$ and $\kappa_{2}$, where we define $\kappa_{1}>\kappa_{2}$ ) (see Fig. 10). We observe that although the two curvatures have similar distributions, the negative curvature $\kappa_{2}$ is more shifted to the left, which results in the negative mean curvature, represented by the capillary pressure in Fig. 9(c) and the negative Gaussian curvature shown in Fig. 9(d). Forming negative and positive principal curvatures is a necessary condition for the oil and water phases to remain continuous in the pore space, implying a structure with many redundant loops [36].

\section{CONCLUSIONS AND FUTURE WORK}

This work has provided pore-scale insights into the dynamics of two-phase fluid flow during water injection in an oil-wet reservoir rock. We have used fast synchrotron x-ray microtomography to visualize the displacement of oil by water and investigate the pore-scale dynamics by examining (i) in situ wettability and displacement contact angles, (ii) pore-filling order, (iii) Haines jumps, (iv) snap-off events, (v) fluid saturations, (vi) specific interfacial areas, (vii) oil-water capillary pressure, and (viii) Gaussian curvature.

We observed that the displacement of oil by water is a drainagelike process. Measurements of local contact angle and the estimation of a value based on energy balance during the displacement confirmed that the medium was oil-wet (water repellent). Hence, the order of pore filling followed an invasion percolation pattern, where throats filled in order of size, with the largest filled first. This is akin to drainage processes in water-wet systems. Water, the nonwetting phase, advances as a connected front displacing oil in the centers of the pore space; oil is confined to movement in wetting layers.

We observed drainage-associated dynamics, e.g., Haines jumps and snap-off, before and after water breakthrough in the imaged rock section. Haines jumps were observed in single and multiple pores, alongside the rearrangement of the nonwetting phase, water, to supply this rapid filling; water retracts from the high-pressure regions (throats) and flows towards regions of low water pressure. Furthermore, the two types of 
snap-off in drainage processes, local and distal snap-off, were observed. In both snap-off events, the trapped (disconnected) water ganglion reaches a new position of capillary equilibrium in the pore space; the water ganglion has a lower absolute capillary pressure than that of the connected water cluster.

The water saturation stabilized to $(38 \pm 2) \%$ after the injection of only 0.58 pore volumes of water; further injection of water did not produce more oil. This was ascribed to the existence of oil in thick connected wetting layers in the corners of the pore space, where the access of water is limited. The oil-wet nature of the surface results in a negative capillary pressure; the Gaussian curvature is also negative, which leads to well-connected oil and water phases in the pore space.

Overall, we have elucidated the invasion patterns and pore-filling events during water injection in a reservoir rock which manifests an oil-wet behavior. The same methods and analysis can be used to characterize signature of two-phase flow dynamics and help design carbon storage, subsurface contaminant transport, fuel cells, batteries, and chemical reactors, for instance, as well as providing benchmark data for the validation and calibration of pore-scale models. Future work can investigate the impact of wettability on flow in different porous media such as soils, packed bed reactors, batteries, fuel cells, and microfluidic devices.

\section{ACKNOWLEDGMENTS}

We acknowledge Abu Dhabi National Oil Company for financially supporting this research. We are very grateful to Ahmed Selem, Catherine Spurin, and Gaetano Garf (Imperial College London) and Christoph Rau, Shashidhara Marathe, Kaz Wanelik, and the staff of Diamond Light Source for their great efforts at the time of the experiment at the synchrotron facility. We further acknowledge Catherine Spurin for her involvement in the design of the experimental research and for performing the investigations.
[1] G. Gompper and D. A. Fedosov, Modeling microcirculatory blood flow: Current state and future perspectives, Wiley Interdiscip. Rev. Syst. Biol. Med. 8, 157 (2016).

[2] P. V. Trusov, N. V. Zaitseva, and M. R. Kamaltdinov, A multiphase flow in the antroduodenal portion of the gastrointestinal tract: A mathematical model, Comput. Math. Methods Med. 2016, 5164029 (2016).

[3] A. Mularczyk, Q. Lin, M. J. Blunt, A. Lamibrac, F. Marone, T. J. Schmidt, F. N. Büchi, and J. Eller, Droplet and percolation network interactions in a fuel cell gas diffusion layer, J. Electrochem. Soc. 167, 084506 (2020).

[4] J. M. Matter and P. B. Kelemen, Permanent storage of carbon dioxide in geological reservoirs by mineral carbonation, Nat. Geosci. 2, 837 (2009).

[5] S. Krevor, M. J. Blunt, S. M. Benson, C. H. Pentland, C. Reynolds, A. Al-Menhali, and B. Niu, Capillary trapping for geologic carbon dioxide storage - From pore scale physics to field scale implications, Int. J. Greenhouse Gas Control 40, 221 (2015).

[6] L. W. Lake, Enhanced Oil Recovery (Prentice Hall, Englewood Cliffs, 1989).

[7] N. Morrow and J. Buckley, Improved oil recovery by lowsalinity waterflooding, J. Petrol. Technol. 63, 106 (2011).

[8] M. J. Blunt, Multiphase Flow in Permeable Media: A Pore-Scale Perspective (Cambridge University Press, Cambridge, 2017).

[9] A. AlRatrout, M. J. Blunt, and B. Bijeljic, Wettability in complex porous materials, the mixed-wet state, and its relationship to surface roughness, Proc. Natl. Acad. Sci. USA 115, 8901 (2018).

[10] C. Sanchez, H. Arribart, and M. M. Giraud Guille, Biomimetism and bioinspiration as tools for the design of innovative materials and systems, Nat. Mater. 4, 277 (2005).

[11] T. Sun, L. Feng, X. Gao, and L. Jiang, Bioinspired surfaces with special wettability, Acc. Chem. Res. 38, 644 (2005).

[12] J. A. Nychka and M. M. Gentleman, Implications of wettability in biological materials science, JOM 62, 39 (2010).

[13] L. Bromberg, X. Liu, I. Wang, S. Smith, K. Schwicker, Z. Eller, and G. K. German, Control of human skin wettability using the
$\mathrm{pH}$ of anionic surfactant solution treatments, Colloids Surf. B 157, 366 (2017).

[14] D. Wilkinson and J. F. Willemsen, Invasion percolation: A new form of percolation theory, J. Phys. A: Math. Gen. 16, 3365 (1983).

[15] R. Lenormand and S. Bories, Description d'un mécanisme de connexion de liaison destiné à l'étude du drainage avec piégeage en milieu poreux, C. R. Acad. Sci. Paris B 291, 279 (1980).

[16] W. B. Haines, Studies in the physical properties of soil. V. The hysteresis effect in capillary properties, and the modes of moisture distribution associated therewith, J. Agr. Sci. 20, 97 (1930).

[17] J. G. Roof, Snap-off of oil droplets in water-wet pores, Soc. Petrol. Eng. J. 10, 85 (1970).

[18] S. Berg et al., Real-time 3D imaging of Haines jumps in porous media flow, Proc. Natl. Acad. Sci. USA 110, 3755 (2013).

[19] M. Andrew, H. Menke, M. J. Blunt, and B. Bijeljic, The imaging of dynamic multiphase fluid flow using synchrotron-based $\mathrm{x}$-ray microtomography at reservoir conditions, Transp. Porous Media 110, 1 (2015.

[20] J. J. Pickell, B. F. Swanson, and W. B. Hickman, Application of air-mercury and oil-air capillary pressure data in the study of pore structure and fluid distribution, Soc. Petrol. Eng. J. 6, 55 (1966).

[21] R. Lenormand, C. Zarcone, and A. Sarr, Mechanisms of the displacement of one fluid by another in a network of capillary ducts, J. Fluid Mech. 135, 337 (1983).

[22] K. Singh, H. Menke, M. Andrew, Q. Lin, C. Rau, M. J. Blunt, and B. Bijeljic, Dynamics of snap-off and pore-filling events during two-phase fluid flow in permeable media, Sci. Rep. 7, 5192 (2017).

[23] M. Rücker et al., From connected pathway flow to ganglion dynamics, Geophys. Res. Lett. 42, 3888 (2015).

[24] S. S. Datta, T. S. Ramakrishnan, and D. A. Weitz, Mobilization of a trapped non-wetting fluid from a three-dimensional porous medium, Phys. Fluids 26, 022002 (2014). 
[25] M. J. Blunt, B. Bijeljic, H. Dong, O. Gharbi, S. Iglauer, P. Mostaghimi, A. Paluszny, and C. Pentland, Pore-scale imaging and modelling, Adv. Water Resour. 51, 197 (2013).

[26] V. Cnudde and M. N. Boone, High-resolution X-ray computed tomography in geosciences: A review of the current technology and applications, Earth Sci. Rev. 123, 1 (2013).

[27] D. Wildenschild and A. P. Sheppard, X-ray imaging and analysis techniques for quantifying pore-scale structure and processes in subsurface porous medium systems, Adv. Water Resour. 51, 217 (2013).

[28] T. Bultreys, W. De Boever, and V. Cnudde, Imaging and imagebased fluid transport modeling at the pore scale in geological materials: A practical introduction to the current state-of-theart, Earth Sci. Rev. 155, 93 (2016).

[29] K. Singh, B. Bijeljic, and M. J. Blunt, Imaging of oil layers, curvature and contact angle in a mixed-wet and a water-wet carbonate rock, Water Resour. Res. 52, 1716 (2016).

[30] T. Pak, I. B. Butler, S. Geiger, M. I. J. van Dijke, and K. S. Sorbie, Droplet fragmentation: 3D imaging of a previously unidentified pore-scale process during multiphase flow in porous media, Proc. Natl. Acad. Sci. USA 112, 1947 (2015).

[31] M. Andrew, B. Bijeljic, and M. J. Blunt, Pore-scale imaging of trapped supercritical carbon dioxide in sandstones and carbonates, Int. J. Greenhouse Gas Control 22, 1 (2014).

[32] M. Kumar, M. A. Knackstedt, T. J. Senden, A. P. Sheppard, and J. P. Middleton, Visualizing and quantifying the residual phase distribution in core material, Petrophysics 51, 10 (2010).

[33] R. T. Armstrong, A. Georgiadis, H. Ott, D. Klemin, and S. Berg, Critical capillary number: Desaturation studied with fast X-ray computed microtomography, Geophys. Res. Lett. 41, 55 (2014).

[34] R. T. Armstrong and S. Berg, Interfacial velocities and capillary pressure gradients during Haines jumps, Phys. Rev. E 88, 043010 (2013).

[35] A. Scanziani, K. Singh, H. Menke, B. Bijeljic, and M. J. Blunt, Dynamics of enhanced gas trapping applied to $\mathrm{CO}_{2}$ storage in the presence of oil using synchrotron $\mathrm{x}$-ray micro tomography, Appl. Energy 259, 114136 (2020).

[36] A. Scanziani, Q. Lin, A. Alhosani, M. J. Blunt, and B. Bijeljic, Dynamics of fluid displacement in mixed-wet porous media, Proc. R. Soc. A 476, 20200040 (2020).

[37] A. R. Kovscek, H. Wong, and C. J. Radke, A pore-level scenario for the development of mixed wettability in oil reservoirs, AIChE J. 39, 1072 (1993).

[38] J. S. Buckley, Effective wettability of minerals exposed to crude oil, Curr. Opin. Colloid Interface Sci. 6, 191 (2001).

[39] Y. Zheng, X. Gao, and L. Jiang, Directional adhesion of superhydrophobic butterfly wings, Soft Matter 3, 178 (2007).

[40] H. Schott, Contact angles and wettability of human skin, J. Pharm. Sci. 60, 1893 (1971).

[41] S. Zhang, J. Huang, Z. Chen, S. Yang, and Y. Lai, Liquid mobility on superwettable surfaces for applications in energy and the environment, J. Mater. Chem. A 7, 38 (2019).

[42] A. Elkhyat, P. Agache, H. Zahouani, and P. Humbert, A new method to measure in vivo human skin hydrophobia, Int. J. Cosmet. Sci. 23, 347 (2001).

[43] A. M. Alhammadi, A. AlRatrout, K. Singh, B. Bijeljic, and M. J. Blunt, In situ characterization of mixed-wettability in a reservoir rock at subsurface conditions, Sci. Rep. 7, 10753 (2017).
[44] See Supplemental Material at http://link.aps.org/supplemental/ 10.1103/PhysRevE.102.023110 for the pore size distribution of the reservoir rock, the table of crude oil properties, a movie of the advancing water front through the pore space, a movie of the oil layer movement during flow, images of oil layer movement during displacement, and a movie of the increasing water saturation in the pore space with time.

[45] NIST Reference Fluid Thermodynamic and Transport Properties Database (REFPROP), version 10, available at https://www. nist.gov/srd/refprop (NIST, Gaithersburg, 2019).

[46] The Engineering ToolBox, Carbon Dioxide - Dynamic and Kinematic Viscosity, available at https://www. engineeringtoolbox.com/carbon-dioxide-dynamic-kinematicviscosity-temperature-pressure-d_2074.html (2020).

[47] T. Jianhua, J. Satherley, and D. J. Schiffrin, Density and interfacial tension of nitrogen-hydrocarbon systems at elevated pressures, Chin. J. Chem. Eng. 1, 223 (1993).

[48] R. A. Salathiel, Oil recovery by surface film drainage in mixedwettability rocks, J. Petrol. Technol. 25, 1216 (1973).

[49] M. Rücker et al., The effect of mixed wettability on porescale flow regimes based on a flooding experiment in Ketton limestone, Geophys. Res. Lett. 46, 3225 (2019).

[50] I. Arganda-Carreras, V. Kaynig, C. Rueden, K. W. Eliceiri, J. Schindelin, A. Cardona, and H. S. Seung, Trainable Weka segmentation: A machine learning tool for microscopy pixel classification, Bioinformatics 33, 2424 (2017).

[51] G. Garfi, C. M. John, S. Berg, and S. Krevor, The sensitivity of estimates of multiphase fluid and solid properties of porous rocks to image processing, Transp. Porous Media 131, 985 (2020).

[52] A. AlRatrout, A. Q. Raeini, B. Bijeljic, and M. J. Blunt, Automatic measurement of contact angle in pore-space images, Adv. Water Resour. 109, 158 (2017).

[53] K. A. Klise, D. Moriarty, H. Yoon, and Z. Karpyn, Automated contact angle estimation for three-dimensional x-ray microtomography data, Adv. Water Resour. 95, 152 (2016).

[54] A. Scanziani, K. Singh, M. J. Blunt, and A. Guadagnini, Automatic method for estimation of in situ effective contact angle from x-ray micro tomography images of two-phase flow in porous media, J. Colloid Interface Sci. 496, 51 (2017).

[55] L. E. Dalton, K. A. Klise, S. Fuchs, D. Crandall, and A. Goodman, Methods to measure contact angles in sc $\mathrm{CO}_{2}$-brine-sandstone systems, Adv. Water Resour. 122, 278 (2018).

[56] M. Khishvand, A. H. Alizadeh, and M. Piri, In-situ characterization of wettability and pore-scale displacements during two- and three-phase flow in natural porous media, Adv. Water Resour. 97, 279 (2016).

[57] N. R. Morrow, Physics and thermodynamics of capillary action in porous media, Ind. Eng. Chem. 62, 32 (1970).

[58] T. Akai, A. Alhammadi, M. Blunt, and B. Bijeljic, Modeling oil recovery in mixed-wet rocks: Pore-scale comparison between experiment and simulation, Transp. Porous Media 127, 393 (2019).

[59] M. J. Blunt, Q. Lin, T. Akai, and B. Bijeljic, A thermodynamically consistent characterization of wettability in porous media using high-resolution imaging, J. Colloid Interface Sci. 552, 59 (2019).

[60] A. Q. Raeini, B. Bijeljic, and M. J. Blunt, Generalized network modeling: Network extraction as a coarse-scale discretization 
of the void space of porous media, Phys. Rev. E 96, 013312 (2017).

[61] H. Dong and M. J. Blunt, Pore-network extraction from microcomputerized-tomography images, Phys. Rev. E 80, 036307 (2009).

[62] C. H. Arns, M. A. Knackstedt, and N. S. Martys, Cross-property correlations and permeability estimation in sandstone, Phys. Rev. E 72, 046304 (2005).

[63] C. H. Arns, M. A. Knackstedt, and K. R. Mecke, Boolean reconstructions of complex materials: Integral geometric approach, Phys. Rev. E 80, 051303 (2009).

[64] H.-J. Vogel, U. Weller, and S. Schlüter, Quantification of soil structure based on Minkowski functions, Comput. Geosci. 36, 1236 (2010).

[65] K. A. Culligan, D. Wildenschild, B. S. B. Christensen, W. G. Gray, M. L. Rivers, and A. F. B. Tompson, Interfacial area measurements for unsaturated flow through a porous medium, Water Resour. Res. 40, W12413 (2004).

[66] R. T. Armstrong, M. L. Porter, and D. Wildenschild, Linking pore-scale interfacial curvature to column-scale capillary pressure, Adv. Water Resour. 46, 55 (2012).

[67] T. Akai, Q. Lin, A. Alhosani, B. Bijeljic, and J. M. Blunt, Quantification of uncertainty and best practice in computing interfacial curvature from complex pore space images, Materials 12, 2138 (2019).

[68] Q. Lin, B. Bijeljic, R. Pini, M. J. Blunt, and S. Krevor, Imaging and measurement of pore-scale interfacial curvature to determine capillary pressure simultaneously with relative permeability, Water Resour. Res. 54, 7046 (2018).

[69] A. Alhosani, A. Scanziani, Q. Lin, Z. Pan, B. Bijeljic, and M. J. Blunt, In situ pore-scale analysis of oil recovery during threephase near-miscible $\mathrm{CO}_{2}$ injection in a water-wet carbonate rock, Adv. Water Resour. 134, 103432 (2019).

[70] M. Andrew, B. Bijeljic, and M. J. Blunt, Pore-by-pore capillary pressure measurements using $\mathrm{x}$-ray microtomography at reservoir conditions: Curvature, snap-off, and remobilization of residual $\mathrm{CO}_{2}$, Water Resour. Res. 50, 8760 (2014).

[71] A. M. Alhammadi, Y. Gao, T. Akai, M. J. Blunt, and B. Bijeljic, Pore-scale $\mathrm{x}$-ray imaging with measurement of relative permeability, capillary pressure and oil recovery in a mixedwet micro-porous carbonate reservoir rock, Fuel 268, 117018 (2020).

[72] K. K. Mohanty, H. T. Davis, and L. E. Scriven, Physics of oil entrapment in water-wet rock, SPE Reservoir Eng. 2, 113 (1987).

[73] T. C. Ransohoff, P. A. Gauglitz, and C. J. Radke, Snap-off of gas bubbles in smoothly constricted noncircular capillaries, AIChE J. 33, 753 (1987).

[74] A. Alhosani, A. Scanziani, Q. Lin, A. Q. Raeini, B. Bijeljic, and M. J. Blunt, Pore-scale mechanisms of $\mathrm{CO}_{2}$ storage in oilfields, Sci. Rep. 10, 8534 (2020).

[75] Q. Lin, B. Bijeljic, S. Berg, R. Pini, M. J. Blunt, and S. Krevor, Minimal surfaces in porous media: Pore-scale imaging of multiphase flow in an altered-wettability Bentheimer sandstone, Phys. Rev. E 99, 063105 (2019). 